\title{
BMJ Open Outbreak response intervention models of vaccine-preventable diseases in humans and foot-and-mouth disease in livestock: a protocol for a systematic review
}

\author{
James M Azam (D) , ${ }^{1}$ Elisha B Are (D) , ${ }^{1}$ Xiaoxi Pang, ${ }^{2}$ Matthew J Ferrari (D) , ${ }^{3}$ \\ Juliet R C Pulliam (1D ${ }^{1}$
}

To cite: Azam JM, Are EB, Pang $X$, et al. Outbreak response intervention models of vaccine-preventable diseases in humans and foot-and-mouth disease in livestock: a protocol for a systematic review. BMJ Open 2020;10:e036172. doi:10.1136/ bmjopen-2019-036172

- Prepublication history and supplemental material for this paper are available online. To view these files, please visit the journal online (http://dx.doi. org/10.1136/bmjopen-2019036172).

Received 04 December 2019 Revised 31 July 2020 Accepted 26 August 2020

Check for updates

(c) Author(s) (or their employer(s)) 2020. Re-use permitted under CC BY-NC. No commercial re-use. See rights and permissions. Published by BMJ.

For numbered affiliations see end of article.

Correspondence to

James M Azam;

jamesazam@sun.ac.za

\section{ABSTRACT}

Introduction Outbreaks of vaccine-preventable diseases continue to threaten public health, despite the proven effectiveness of vaccines. Interventions such as vaccination, social distancing and palliative care are usually implemented, either individually or in combination, to control these outbreaks. Mathematical models are often used to assess the impact of these interventions and for supporting outbreak response decision making. The objectives of this systematic review, which covers all human vaccine-preventable diseases, are to determine the relative impact of vaccination compared with other outbreak interventions, and to ascertain the temporal trends in the use of modelling in outbreak response decision making. We will also identify gaps and opportunities for future research through a comparison with the foot-and-mouth disease outbreak response modelling literature, which has good examples of the use of modelling to inform outbreak response intervention decision making.

Methods and analysis We searched on PubMed, Scopus, Web of Science, Google Scholar and some preprint servers from the start of indexing to 15 January 2020. Inclusion: modelling studies, published in English, that use a mechanistic approach to evaluate the impact of an outbreak intervention. Exclusion: reviews, and studies that do not describe or use mechanistic models or do not describe an outbreak. We will extract data from the included studies such as their objectives, model types and composition, and conclusions on the impact of the intervention. We will ascertain the impact of models on outbreak response decision making through visualisation of time trends in the use of the models. We will also present our results in narrative style.

Ethics and dissemination This systematic review will not require any ethics approval since it only involves scientific articles. The review will be disseminated in a peer-reviewed journal and at various conferences fitting its scope. PROSPERO registration number CRD42020160803.

\section{INTRODUCTION}

Great progress has been made globally in reducing the high rates of child mortality and
Strengths and limitations of this study

- To the best of our knowledge, this is the first systematic review to examine studies that use mechanistic models to assess the relative benefit of vaccination compared with other outbreak interventions, and to ascertain the impact of modelling studies on policy making and decision making.

- The detailed search strategy used in this systematic review captures all human vaccine-preventable diseases.

- This review protocol is developed according to the Preferred Reporting of Items in Systematic Reviews and Meta-Analyses guidelines, hence, reported in a standard manner.

- This review will only consider studies published in English and may miss any studies written in other languages, but our initial search results show that only a few relevant studies were published in nonEnglish languages.

morbidity attributed to vaccine-preventable diseases. ${ }^{1}$ However, outbreaks of these diseases continue to threaten global health and wellbeing. When these outbreaks occur, outbreak response interventions may be organised to control or halt disease spread. There are numerous interventions for preventing and controlling outbreaks of vaccine-preventable diseases. Immunisation is one of the most cost-effective. $^{2}$ Additionally, a diversity of other interventions exist for complementing vaccination, but their implementation depends on the disease type, epidemic size, intervention timing and budget allocation. ${ }^{3}$ For instance, during outbreaks of diseases like smallpox and Ebola, a combination of contact tracing, isolation, quarantine and vaccination have been employed to effectively control the pathogen. ${ }^{4-6}$ More generally, case 
management and vaccination are used concurrently to reduce transmission and disease-related mortality during outbreaks of vaccine-preventable diseases. ${ }^{7}$

Outbreak response interventions have many public health and economic benefits. Vaccination particularly helps increase population-level immunity, preventing illness and death, and reduces productivity losses due to illness. ${ }^{8}$ For outbreaks of diseases like measles, whose control through vaccination is part of the routine immunisation schedule, outbreak response vaccination campaigns serve as an opportunity to immunise individuals who were missed by routine vaccination. ${ }^{9}$

Mathematical models are useful for understanding many aspects of outbreaks. ${ }^{10-12}$ Particularly, outbreak response intervention models are an application of mathematical models for studying efficient ways of controlling outbreaks. They have three general applications, namely forecasting of epidemic spread, analysing of disease surveillance, and assessment of intervention impact. ${ }^{13}$ They are widely employed for investigating the potential impact of reactive interventions, identifying and assessing strategies that help achieve efficient interventions, and considering future intervention decisions. ${ }^{12}{ }^{14}$ Over the past few decades, there has been a rise in the use of outbreak response intervention models for informing response strategies, decision making, and policy making. ${ }^{11}{ }^{15}$ In fact, a recent theme issue by the Philosophical Transactions of the Royal Society acknowledged this rise in their use and highlighted some current modelling work with regard to our understanding and control of outbreaks of infectious diseases of humans, animals, and plants. ${ }^{13}$ However, we are unaware of any systematic review that has examined this increase in trend for all human vaccine-preventable diseases. Moreover, it is common for models to be described in the literature as being useful for informing outbreak response decision making, ${ }^{16}$ but to the best of our knowledge, no systematic review has evaluated the degree to which this assertion is true. Hence, an objective of this review will be to assess whether models are increasingly being used to inform outbreak response decision making and policy making.

It is clear in the outbreak response literature that a wealth of policy-relevant models have amassed from previous efforts to control outbreaks of foot-and-mouth disease (FMD) ${ }^{17-19}$ In fact, models of foot-and-mouth disease were the first to be used for outbreak response decision making. ${ }^{16} \quad 20$ Additionally, FMD outbreak response models are well studied in epidemiological modelling and are often used to illustrate the usefulness of models in outbreak response decision making. ${ }^{21}$ We will, therefore, include eligible FMD modelling studies to help us to compare the current practice of outbreak response modelling for intervention impact assessment and decision making in the human vaccine-preventable diseases literature.

Several systematic reviews have been conducted to describe the use of models to assess the impact of interventions on outbreaks of infectious diseases and to ascertain their impact on policy making and decision making. ${ }^{2022}$ However, these reviews are often focused on a few diseases. One systematic review, for instance, explored models that assessed the impact of future vaccines on tuberculosis (TB) infection. ${ }^{23}$ Additionally, few reviews have attempted to assess the conclusions of models on the relative benefit of vaccination compared with other outbreak interventions during outbreaks of human vaccine-preventable diseases. For example, a systematic review by Lee $e t a l^{24}$ compared the effectiveness of combination strategies with single strategies but the baseline intervention was not vaccination and the disease scope was pandemic influenza. Hence, this systematic review will identify the overall conclusion on the relative impact of vaccination compared with other outbreak interventions, when models are used as the assessment tool. This systematic review will also highlight research gaps and opportunities for future research. The main objectives for this review are informed by that of a larger project, which involves the formulation and use of models to evaluate alternative intervention strategies for responding to measles outbreaks. This review will be useful to infectious disease modellers, both novice and expert, and policy makers who may already be using or considering the use of models for decision making.

\section{Objectives}

Our main objectives are:

1. To assess the relative impact of vaccination compared with other reactive interventions during outbreaks of human vaccine-preventable diseases.

2. To determine whether mathematical modelling is increasingly impacting on the policy making and decision making process during outbreak response.

Additionally, our secondary objectives are:

1. To summarise similarities and differences in modelling approaches of included studies.

2. To identify knowledge gaps in modelling approaches and opportunities for advancement.

3 . To identify and summarise parallels and contrasts between the outbreak response modelling literature for vaccine-preventable diseases in humans, and foot-andmouth disease in livestock.

\section{METHODS}

In conducting this review, we will adhere to the criteria listed in the Preferred Reporting of Items in Systematic Reviews and Meta-Analyses statement. ${ }^{25}{ }^{26}$ A supplementary file contains the populated checklist for the protocol (see online supplemental file 1).

For this systematic review, we will consider a model as mechanistic if it describes the disease's individual-level or population-level transmission dynamics by capturing its biological mechanisms or natural history with some form of mathematical equation ${ }^{22} 27$. Consequently, we describe as outbreak response intervention models, all mechanistic models that have been developed to investigate the 


\begin{tabular}{|c|c|}
\hline Cholera & Mumps \\
\hline Dengue & Pertussis \\
\hline Diphtheria & Pneumococcal disease \\
\hline Hepatitis A & Poliomyelitis \\
\hline Hepatitis B & Rabies \\
\hline Hepatitis E & Rotavirus \\
\hline Haemophilus influenzae type b & Rubella \\
\hline Human papillomavirus & Tetanus \\
\hline Influenza & Tickborne encephalitis \\
\hline Japanese encephalitis & Tuberculosis \\
\hline Malaria & Typhoid \\
\hline Measles & Varicella \\
\hline Meningococcal meningitis & Yellow fever \\
\hline
\end{tabular}

impact of any intervention to the outbreak of a vaccinepreventable disease affecting humans.

\section{Patient and public involvement}

This research will not require the involvement of patients as the review will involve the use of secondary information collected from modelling studies.

\section{Eligibility criteria}

Here, we describe the criteria for article selection.

\section{Type of studies}

We will consider studies containing a mathematical model, which is mechanistic based on our earlier definition, and is used for assessing vaccination and/or other interventions mounted during an outbreak of any of the human vaccine-preventable diseases listed in table 1 below. Table 1 contains WHO's published list of human vaccine-preventable diseases. ${ }^{28}$ Even though Ebola is not on the list provided by $\mathrm{WHO}$, we will include it in our search because there is a vaccine, which has been used for outbreak response in Central, East and West Africa ${ }^{29}$ and has been modelled in the literature.

We will limit the studies to those published in English. For the search period restriction, the beginning date limit will be based on how far back the database can be searched and the upper limit will be 15 January 2020 .

\section{Type of intervention}

We will consider outbreak response vaccination and other outbreak interventions, that is, any responses mounted because of an outbreak, such as social/physical distancing, quarantine, isolation, palliative care, media coverage /information campaigns, education and others indicated in the articles.

\section{Outcomes}

The two main outcomes will be a conclusion on the temporal trends in the use of modelling as a decisionmaking tool during outbreak response of human vaccine-preventable diseases, and the overall conclusion on the relative benefit of vaccination and non-vaccination interventions mounted in response to outbreaks of human vaccine preventable diseases, with modelling as the tool of assessment. The secondary outcomes will include a summary of the outbreak response modelling landscape. We will obtain this in terms of the diseases and interventions studied, classes of models used, mathematical or statistical approaches for incorporating the intervention(s), and method used to analyse/evaluate the model and intervention. Other outcomes will be the types of equations used, the conclusions drawn from the models, study limitations stated and recommendations provided.

\section{Information sources}

We will search through the following sources:

1. Bibliographic databases: Scopus, PubMed and Web of Science.

2. Preprint: bioRxiv.org, and medRxiv.org.

3. Grey literature: Google Scholar.

\section{Search strategy}

With feedback from the Stellenbosch University Faculty of Science Librarian, we have developed search strings for the three bibliographic databases and Google Scholar. Details of the search strings can be found in the online supplemental file 2 . To validate the search string, we used a list of known references from the literature and found that the strings capture all the relevant articles.

Preprint servers do not support Boolean searches, making it difficult to predefine the exact search procedure. We will, therefore, hand search the preprint servers with keywords such as 'outbreak response', 'model', and their synonyms. The final procedure will be reported in the systematic review.

To identify relevant grey literature, we will search through Google Scholar, which supports Boolean searches, and websites of epidemic response organisations that are known (or likely) to use modelling in understanding outbreaks, for example, the Centers for Disease Control and Prevention. We will also contact authors from cited unpublished literature in the studies we will identify from the peer-reviewed and preprint literature.

\section{STUDY RECORDS}

\section{Data management}

The initial search results will be imported into EndNote X7.8 (endnote.com) for deduplication. Following that, the Rayyan web tool ${ }^{30}$ will be used for the study selection. The KoboToolbox web tool (https://www.kobotoolbox. $\mathrm{org} /$ ) will be used to extract the data from included studies. The extracted data will be exported in a commaseparated values format for further analyses. All postprocessing of the exported data, including visualisations, will be performed with the $\mathrm{R}$ language. ${ }^{31}$ 


\section{Selection process}

In the first stage, one reviewer will examine the preprints and grey literature search results to check whether any have been published as peer-reviewed articles. The reviewer will achieve this using the author names and working titles. If any of such exist, the reviewer will remove the preprint/grey literature version from the search results and record the number of removed records. If any uncertainties arise, the reviewer will consult the other reviewers. Following that, the reviewer will remove the duplicates from the total resulting records, using EndNote X7.8. With the aid of the Rayyan web tool the reviewers will screen the titles and abstracts, and if necessary, full text of resulting articles in duplicate using the inclusion/exclusion criteria listed below.

\section{Inclusion}

1. Diseases are either listed in table 1, Ebola or foot-andmouth disease.

2. Mathematical modelling studies.

3. The mathematical model is mechanistic, that is, its structure is represented with at least one mathematical equation informed by explicit assumptions about the natural history of the disease. ${ }^{22}$

4. The modelling study assesses the impact of an intervention during an outbreak of one of the eligible diseases.

5. The study is written in English.

\section{Exclusion}

1. Reviews, whether peer-reviewed or not.

2. Not a human vaccine-preventable disease listed in table 1, Ebola or foot-and-mouth disease.

3. Not describing an outbreak.

4. Does not formulate or use a model.

5. Model is not mechanistic according to our definition above.

6. Not written in English.

7. Full-text unobtainable after contacting the school librarian, and the corresponding author.

\section{Data collection process}

We will develop a data extraction form according to the items in online supplemental file 3 . The reviewers will initially pilot the form with an article on each of the distinct diseases from the included articles to resolve any confusion. The pilot phase will help ensure we capture any form of non-standard practice across the various disease models. Following that, the reviewers will split the data collection task among themselves and work independently. We will combine the resulting data after a set number of articles, and clarify any confusions further encountered, through discussion.

\section{DATA ITEMS}

Three reviewers will independently extract the data from their share of included articles according to the data items outlined in the online supplemental file 3 provided. If any disagreements arise from the data extraction process, we will resolve it through discussions with the other two reviewers.

\section{QUALITY ASSESSMENT}

It is not the objective of this systematic review to assess the quality of the included models or to select a best or worst model or model design. We will, therefore, not be assessing the quality of the included modelling studies.

\section{DATA}

\section{Synthesis}

We will report in a narrative style, comparing groups of articles sharing common approaches and themes. The themes will include diseases modelled, classes of models, categories of objectives, and so forth. For example, we will compare which articles employed deterministic models vs stochastic models. These groupings will also be summarised in a citation table. In addition, we will study the included studies from the outbreak response modelling literature for foot-and-mouth disease in livestock, and the human vaccine-preventable disease outbreak modelling literature to highlight their commonalities and differences in approach, objectives, and so on. This will help highlight any gaps and opportunities as well as recommendations we will provide as an outcome of this review for the human vaccine-preventable disease outbreak response modelling community.

\section{ETHICS AND DISSEMINATION}

This study does not require any ethics approval as we will not be collecting any primary data. We will disseminate our results through a peer-reviewed journal and conferences.

\section{Author affiliations}

${ }^{1}$ South African DSI-NRF Centre of Excellence in Epidemiological Modelling and Analysis (SACEMA), Stellenbosch University, Cape Town, Western Cape, South Africa ${ }^{2}$ Department of Mathematics, The University of Manchester, Manchester, UK ${ }^{3}$ Center for Infectious Disease Dynamics, Department of Biology, The Pennsylvania State University, University Park, Pennsylvania, USA

Twitter James M Azam @james_azam

Acknowledgements We are thankful to the Stellenbosch University Faculty of Science Librarian, Marie Theron, for her feedback in improving the search strategy.

Contributors JMA is the guarantor of this manuscript. JMA and JRCP conceived the study. JMA prepared the manuscript with the thorough intellectual feedback from JRCP, MJF, XP and EBA. All authors read and approved the final copy of the manuscript.

Funding JMA is being funded with a South African DSI-NRF Centre of Excellence in Epidemiological Modelling and Analysis (SACEMA) PhD bursary with grant number 44895.

Competing interests None declared.

Patient and public involvement Patients and/or the public were not involved in the design, or conduct, or reporting, or dissemination plans of this research.

Patient consent for publication Not required. 
Provenance and peer review Not commissioned; externally peer reviewed.

Open access This is an open access article distributed in accordance with the Creative Commons Attribution Non Commercial (CC BY-NC 4.0) license, which permits others to distribute, remix, adapt, build upon this work non-commercially, and license their derivative works on different terms, provided the original work is properly cited, appropriate credit is given, any changes made indicated, and the use is non-commercial. See: http://creativecommons.org/licenses/by-nc/4.0/.

\section{ORCID iDs}

James M Azam http://orcid.org/0000-0001-5782-7330

Elisha B Are http://orcid.org/0000-0002-0710-7607

Matthew J Ferrari http://orcid.org/0000-0001-5251-8168

Juliet R C Pulliam http://orcid.org/0000-0003-3314-8223

\section{REFERENCES}

1 Machingaidze S, Wiysonge CS, Hussey GD. Strengthening the expanded programme on immunization in Africa: looking beyond 2015. PLoS Med 2013;10:1-5.

2 Centers for Disease Control and Prevention (CDC). Vaccine preventable deaths and the global immunization vision and strategy, 2006-2015. MMWR Morb Mortal Wkly Rep 2006;55:511-5.

3 Keeling MJ, Rohani P. Modeling infectious diseases in humans and animals, 2007. Available: http://www.sciencedirect.com/science/ article/B98GG-4T7XCGF-9D/2/95c40489e0e5ee3c0670ee1cf3f0e65f

4 Ajelli M, Parlamento S, Bome D, et al. The 2014 Ebola virus disease outbreak in Pujehun, Sierra Leone: epidemiology and impact of interventions. BMC Med 2015;13:1-8.

5 Chowell G, Kiskowski M. Modeling ring-vaccination strategies to control Ebola virus disease epidemics. In: Mathematical and statistical modeling for emerging and re-emerging infectious diseases. Springer International Publishing, 2016: 71-87.

6 Porco TC, Holbrook KA, Fernyak SE, et al. Logistics of community smallpox control through contact tracing and ring vaccination: a stochastic network model. BMC Public Health 2004;4:34.

7 Klepac P, Bjørnstad ON, Metcalf CJE, et al. Optimizing reactive responses to outbreaks of immunizing infections: balancing case management and vaccination. PLoS One 2012;7:e41428.

8 Stack ML, Ozawa S, Bishai DM, et al. Estimated economic benefits during the "decade of vaccines" include treatment savings, gains in labor productivity. Health Aff 2011;30:1021-8.

9 World Health Organization. Measles vaccines: WHO position paper, April 2017 - Recommendations. Vaccine 2019;37:205-27.

10 Willem L, Verelst F, Bilcke J, et al. Lessons from a decade of individual-based models for infectious disease transmission: a systematic review (2006-2015). BMC Infect Dis 2017;17:612.

11 Lofgren ET, Halloran ME, Rivers CM, et al. Opinion: mathematical models: a key tool for outbreak response. Proc Natl Acad Sci U S A 2014;111:18095-6.
12 Elizabeth Halloran M, Levin BR. Infectious diseases of humans: dynamics and control (pbk EDN). Trends Microbiol 1993;1:202-3.

13 Thompson RN, Brooks-Pollock E. Preface to theme issue 'Modelling infectious disease outbreaks in humans, animals and plants: epidemic forecasting and control'. Philos Trans $R$ Soc Lond B Biol Sci 2019;374:20190375.

14 Mishra S, Fisman DN, Boily M-C. The ABC of terms used in mathematical models of infectious diseases. J Epidemiol Community Health 2011;65:87-94.

15 Thompson RN, Brooks-Pollock E. Detection, forecasting and control of infectious disease epidemics: modelling outbreaks in humans, animals and plants. Philos Trans $R$ Soc Lond B Biol Sci 2019;374:20190038.

16 Keeling MJ. Models of foot-and-mouth disease. Proc Biol Sci 2005;272:1195-202.

17 Keeling MJ, Woolhouse MEJ, May RM, et al. Modelling vaccination strategies against foot-and-mouth disease. Nature 2003;421:136-42.

18 Roche SE, Garner MG, Sanson RL, et al. Evaluating vaccination strategies to control foot-and-mouth disease: a model comparison study. Epidemiol Infect 2015;143:1256-75.

19 Hayama Y, Yamamoto T, Kobayashi S, et al. Mathematical model of the 2010 foot-and-mouth disease epidemic in Japan and evaluation of control measures. Prev Vet Med 2013;112:183-93.

20 Louz D, Bergmans HE, Loos BP, et al. Emergence of viral diseases: mathematical modeling as a tool for infection control, policy and decision making. Crit Rev Microbiol 2010;36:195-211.

21 Keeling MJ, Rohani P. Modeling infectious diseases in humans and animals. Princeton University Press, 2011: 1-368.

22 Lessler J, Cummings DAT. Mechanistic models of infectious disease and their impact on public health. Am J Epidemiol 2016;183:415-22.

23 Harris RC, Sumner T, Knight GM, et al. Systematic review of mathematical models exploring the epidemiological impact of future TB vaccines. Hum Vaccin Immunother 2016;12:2813-32.

24 Lee VJ, Lye DC, Wilder-Smith A. Combination strategies for pandemic influenza response - a systematic review of mathematical modeling studies. BMC Med 2009;7:76.

25 Moher D, Liberati A, Tetzlaff J, et al. Preferred reporting items for systematic reviews and meta-analyses: the PRISMA statement. PLoS Med 2009;6:e1000097.

26 Liberati A, Altman DG, Tetzlaff J, et al. The PRISMA statement for reporting systematic reviews and meta-analyses of studies that evaluate health care interventions: explanation and elaboration. PLoS Med 2009;6:e1000100-4.

27 Reiner RC, Perkins TA, Barker CM, et al. A systematic review of mathematical models of mosquito-borne pathogen transmission: 1970-2010. J R Soc Interface 2013;10:20120921.

28 WHO. Vaccines and diseases. immunization, vaccines and biologicals, 2016

29 Venkatraman N, Silman D, Folegatti PM, et al. Vaccines against Ebola virus. Vaccine 2018;36:5454-9.

30 Ouzzani M, Hammady H, Fedorowicz Z, et al. Rayyan-a web and mobile APP for systematic reviews. Syst Rev 2016;5:210.

31 R Core Team. R: a language and environment for statistical computing, 2020. Available: https://www.r-project.org/ 